\title{
New Possibilities of Complex Forensic Analysis in the Field of Cultural Heritage Protection and Counterfeits Identification
}

\author{
Marek Kotrlýl, ${ }^{1}$ and Ivana Turková ${ }^{1}$ \\ 1. Institute of Criminalistics Prague (ICP), Czech Republic. \\ 2. Charles University in Prague, Faculty of Science, Praha, Czech Republic. \\ * Corresponding author: marek.kotrly@pcr.cz
}

The current world market is flooded with counterfeits in each branch and market with objects of art make no difference. Most of the estimates agree that approximately 30\% of items on the art market are counterfeits. Very often it is the modern art or contemporary artists where it is easier to put the pieces on the market. Unfortunately, also identification of these counterfeits is more difficult if they are of a good quality.

In the forensic field, the tendency is to apply a complex analytical approach enabling to analyze all components of a piece of art. The area of paintings analysis is most probably the broadest. The complex analyses of the colour layer and other materials carried out can be generally divided into the totally nondestructive and those requiring a microsampling.

Methods ranking between the non-destructive analyses are initial imaging in visible spectrum, infrared and UV spectrum, multispectral photography and x-ray imaging. The x-ray imaging is used for nondestructive detection of composition, structure and condition of pieces of art, for identification of repaintings and underpaintings and for identification of differences in style between originals and counterfeits. Other non-destructive methods include mobile XRF and Raman spectroscopy All these methods are usually used as the screening ones to obtain the initial information about the examined item and to specify the locations for collecting microsamples necessary for further analysis.

A whole range of devices and techniques are used for further instrumental analysis. The most important are:

a) optical and electron microscopy and microanalysis (SEM/FIB and EDS/WDS, mXRF) - basic methods applied for most inorganic samples. Their advantage is non-destructivity, the sample can be further used. If it is not necessary to carry out examination from a cross-section of colour layers, it is possible to insert the item in the microscope chamber directly without any adjustment (size up to 20 by $20 \mathrm{~cm}$ ). For many items, there is no other possibility - gemstones, jewellery, etc. To determine quantitative characteristics (for example morphological parameters of mineral grains), methods of imaging analysis are used. The data of individual samples are compared with each other using multivariate statistical analyzes To compare textile fibres and their fragments, methods of quantitative colour measurement in a microscale are used. To study microelements and admixtures, an XRF device built directly in the SEM chamber (mXRF) with the spot size $30 \mu \mathrm{m}$ is used with benefits[1]. The device enables also a parallel EDS/XRF analyses and is successfully used to analyse trace elements for example in the synthetic gemmological materials which imitate natural gemstones. Based on the microelements content, it is possible to specify for example the origin of cubic zirconia (SiC), etc. The FIB methods are applied for example to study thin layers and metal material structure (e.g. imitations of gold items covered by titanium nitride) [2]. The items are evaluated in a complex way and also canvases are 
examined - a type of weave is specified, fibres are identified and fibre pigments and dyes are analysed. Material the frames are made of is examined, etc.

b) x-ray powder diffraction and microdiffraction (XRD, mXRD) - enable direct phase analysis of substances even in a mixture. The mXRD allows to obtain full structure information from the area of approximately $100 \mu \mathrm{m}$ and so it is possible to analyse directly the individual pigment grains, thin layers, etc.

c ) infrared spectroscopy (IR, FTIR) and Raman spectroscopy - important complementary methods. A problem can appear concerning mixtures analysis when very significant overlay of absorption belts occurs. A problem concerning the IR is the creation of so called mirror reflections which originate very often in spectra from polished sections. When talking about the Raman spectroscopy, it is the fluorescence which can totally disable measurement for the used laser wave length.

d) gas chromatography with mass detection (GC-MS, MS/MS, etc.) - these are used especially to prove organic substances in mixtures. In paintings counterfeits, they are used to identify the content of impregnants using for artificial ageing.

From the other methods, it is possible to name for example botanical methods (specification of kinds of wood), biological methods or genetic analysis (analysis of binders of a biological origin, etc.). Also a signature analysis is carried out by standard forensic handwriting technique. However, it is very difficult as it usually does not represent a bound font and the texts are very short.

The multidisciplinary approach enabled to collect a large set of data both for the art material database and for the database of painting techniques of important Czech painters. The research using mobile spectroscopic methods brought very satisfactory results. Thanks to them it was possible to carry out a detailed characterization of the individual authors' pallets. The obtained data are fundamental for the authenticity assessment of works of art. All the data obtained, including the primary documentation, are saved in a specifically programmed databases which shall be later used for paintings authenticity assessment. The databases are at an initial stage nevertheless they contain more than eleven thousand data entries.

In practice, a few dozens of cases is processed a year. Big cases contain even a few hundred items. Besides the pigment and paintings, colour layers analysis and comparison in a wider sense, also a significantly wider spectrum of other materials is examined. Let us name a complex analysis of samples of petrology and mineralogy character (e.g. relicts of statues, sculptures, gemmology items, etc.), analyses of metal materials in a larger sense, fibres specification and textile material analysis, analysis of tool marks (e.g. on painting frames, comparison with paintings, detection of tool marks, etc.), etc [4].

\section{References:}

[1] M Kotrlý and I Turková, Proc. SPIE 9486 (2015), p. 948614.

[2] M Kotrlý, Microsc. Microanal. 23 (S1) (2017), p. 256.

[3] M Kotrlý, Microsc. Microanal. 22 (S3) (2016), p. 2032.

[4] Acknowledgements - Microanalytical methods at ICP has been supported by the projects of the Ministry of the Interior of the Czech Republic: VI20152020035, VI20172020050. 